\title{
Challenges on the Follow-Up Experimental Leptospiral Infection in Sheep
}

\author{
Matheus Costa da Rosa', Bruno Ribeiro Rocha ${ }^{2}$, Lucas Correia ${ }^{2}$, \\ Gabriel Martins'2, Odir Antônio Dellagostin' ${ }^{1}$ \& Walter Lilenbaum²
}

\begin{abstract}
Background: Leptospirosis is currently a source of significant economic losses in the agribusiness; as such, experimental studies on this infection are required to develop a better understanding of the pathogenesis, treatment, and immunoprophylaxis of the disease. Sheep may represent a good model for ruminants in such models. Despite the extent of the studies that has been conducted thus far, researchers have yet to reach a consensus on the experimental practices to apply for leptospirosis in this animal species, and several gaps in understanding remain. To bridge these gaps, the present study aimed to assess the usage of several tools for the monitoring of experimental leptospirosis in sheep.

Materials, Methods \& Results: Twelve Santa Ines sheep of different ages were each allocated to one of four groups (A, B, $\mathrm{C}$, and D). The subjects in groups A, B, and C received different doses of Leptospira interrogans serogroup Icterohemorrhagiae by intraperitoneal route, $1 \times 10^{2}, 1 \times 10^{5}$, and $1 \times 10^{8}$ respectively. Group D was the control. Hematological, biochemical and clinical parameters were evaluated daily. Serology by microscopic agglutination test (MAT) and PCR were performed to evaluate the infection status. The most remarkable clinical signs were fever $\left(41^{\circ} \mathrm{C}\right)$ and dehydration, and acute pain (cub). Two animals from Group C presented leukocytosis. Only those in Group C exhibited positive results according to serology, while positivity in PCR was observed in animals in groups A and C. The results of the experiment indicated that sheep may be experimentally infected and can, therefore, be used as a model for leptospirosis in ruminants. Clinical signs cannot be considered to represent a reliable parameter for evaluating the development of leptospirosis in experimentally infected sheep. We recommend the use of urine PCR and serology to confirm the infection in experimentally infected animals and daily complete blood count (CBC) as a follow-up tool.

Discussion: It was observed that the clinical signs cannot be considered as a reliable parameter to evaluate the pathogenesis in experimentally infected ewes, being important to emphasize that the age of the animals does not seem to alter their susceptibility to the infection. This finding is in agreement with other experimental studies, which report that leptospirosis infection in ruminants occurs asymptomatic and subclinical. Hematological and biochemical tests proved to be adequate tools to monitor the experimental infection. Studies have shown that the complete blood count has been used to monitor the acute phase of leptospirosis and is effective in detecting anemia and leukocytosis with neutrophilia in ruminants. Despite the lack of clinical signs, the serological and molecular results confirmed the experimental infection. PCR has been used as an important tool in the diagnosis of leptospirosis. In addition, the current study is the first of its kind to use PCR to detect the carrier status in experimentally infected ewes. Despite this limitation, PCR was very effective in confirming the infection and should be considered for use in experimental studies. Sheep have been used as a good experimental model in several studies, sheep are relatively small compared to other ruminants and can be easily allocated in smaller pens and pens, facilitating the management of research and minimizing the costs of experimentation. In this context, we suggest that sheep represent a good model for the study of leptospirosis in ruminants and therefore a reliable protocol for experimental infection by leptospirosis is necessary.
\end{abstract}

Keywords: ruminants, Leptospira, experimental infection, animal model. 


\section{INTRODUCTION}

Leptospirosis is an infectious, zoonotic disease caused by pathogenic spirochetes of the genus Leptospira [21]. This disease causes significant economic losses to livestock [24,12], mainly due to abortions, premature births, stillbirths and weak calf syndrome [5]. In ruminants, leptospirosis usually presents as a subclinical and silent infection [23]. Despite the significance of this disease, there is a lack of available information on the relationship between infection of leptospires and their hosts, particularly in small ruminants, and few studies have been conducted with the intention of developing a better understanding of infectious aspects such as pathogenesis, vaccinology and immune response $[11,14]$.

The pioneers in the experimental sheep infection were Langham et al. [11], who infected ten sheep of 4-5 months of age with a $L$. interrogans serovar Pomona strain and observed acute signs of infection, such as anorexia, jaundice and hematuria. A similar study conducted at a later date was the first to report the concentration of $1 \times 10^{8}$ leptospires [10]. A few years later, some experimental studies focused on the use of adapted strains, such as the Hardjo [2,9,14]. Due to the lack of updating of the few studies already done, particularly in our scenario, in order to have a better understanding of the infectious aspects in small ruminants, several gaps still need to be elucidated, such as the infectious dose, strains, the route of infection and the methods to follow the experimental infection. Considering the importance of these animals as models, the purpose of the present study was to assess the usage of several tools for the monitoring of experimental leptospirosis in sheep.

\section{MATERIALS AND METHODS}

\section{Animals and experimental groups}

The clinical trial used 12 sheep of the Santa Ines breed, the most common breed in Brazil. Animals were randomly allocated into 4 groups of 3 animals each. Three groups were experimentally infected (A, $\mathrm{B}$, and C) and one remained as the control (D). Each group consisted of one cub (4-6 months old), one young adult (1-year-old), and one mature adult (4-year-old). There was no contact between animals from different groups. Water, food supply, and excreta collection were isolated to avoid cross infection among groups.
The experiments were carried out over a period of 14 days. After that, the animals were all intramuscularly treated with Penfort PPU ${ }^{1}$ [streptomycin $(25 \mathrm{mg} / \mathrm{kg}$ ) and penicillin (25,000 IU)] for 3 days, independently of the outcomes. After treatment, the animals were released only after exhibiting PCR negative results.

\section{Experimental infection}

Prior to the beginning of the experiment, blood (serology) and urine (PCR) samples were collected to confirm the absence of a preexistent infection. Experimental animals received different doses of low-passage Leptospira interrogans, serogroup Icterohaemorrhagiae, strain FIOCRUZ L1-130 by intraperitoneal route, according to the method described by Andreani et al. [2]. Groups A, B, and C received $1 \times 10^{2}, 1 \times 10^{5}$, and $1 \times 10^{8}$ of leptospires respectively. Group D (the control) received $1 \mathrm{ml}$ of sterile culture medium $(\mathrm{EMJH})$.

\section{Sampling}

Blood samples were collected daily for 14 days via puncture of the jugular vein using sterile needles $(40 \times 12 \mathrm{~mm})$ and were stored in two vacuum tubes ${ }^{2}$ (Vacutainer®). One tube (without anticoagulant) was allowed to clot, and the serum was used for serology and biochemistry, while the other (with EDTA) was used to assess the complete blood count (CBC). Serum aliquots were stored in duplicates in $1.5 \mathrm{~mL}$ micro tubes at a temperature of $-20^{\circ} \mathrm{C}$ until processing. Urine samples were collected after the intravenous administration of furosemide $5 \mathrm{mg} / \mathrm{kg}$. Urine was collected at three-day intervals (D0, D3, D6, D9, D12, and D14) and stored in sterile conical tubes $(\sim 15 \mathrm{~mL})$. Aliquots were transferred to micro tubes $(1.5 \mathrm{~mL})$ with $100 \mu \mathrm{L}$ of phosphate buffer, identified, and stored at $-4^{\circ} \mathrm{C}$ until processing.

\section{Clinical signs}

Pyrexia, prostration, dorsal arches (indicative of pain), jaundice, hematuria, dyspnea, polypnea, dehydration, and color of mucosae were evaluated daily by the same veterinarian.

\section{Serology}

To detect anti-Leptospira antibodies, a microscopic agglutination test (MAT) was performed using an antigen panel that consisted of 28 reference strains (Institut Pasteur) ${ }^{4}$ in accordance with international standards (OIE, 2014). Sera displaying at least 50\% agglutinating activity at a 1:100 dilution were considered reactive. 


\section{Urinary PCR}

Leptospiral DNA was extracted by the Wizard SV Genomic DNA Purification System ${ }^{\circledR}{ }^{5}$. PCR targeting the lipL32 gene was performed as described by Hamond et al. [8].

\section{Hematological and biochemistry analyses}

Hematological patterns were determined according to the methods proposed by Salviano et al. [22]. Globular volume was determined by the microhematocrit method, and plasma protein and fibrinogen were evaluated [26]. Blood aliquots were stained with TÜRK's solution, and cells were counted in Neubauer's chamber [20]. Specific leukogram was performed by fast dye staining and counting (InstantProv $\left.{ }^{\circledR}\right)^{6}$.

Concentration of urea and creatinine; seric activity of alanine aminotransferase (ALT); alkaline phosphatase (FAL) and gammaglutamyltransferase (GGT); total protein and fractions (albumin and globulin); and total, direct and indirect bilirubin was analyzed by spectrophotometry in accordance with the manufacturer's instructions (Labtest $\left.{ }^{\mathrm{TM}}\right)^{7}$.

\section{RESULTS}

\section{Clinical signs}

After D7 post-infection (p.i.), 5 animals (55.5\%) presented hypochromic mucosa, which could be considered to be a mild unspecific clinical sign of infection. The possible anemia of those animals was not confirmed at CBC. The most remarkable clinical signs, which could clearly be related to the experimental infection, were observed in two animals in Group C, a young animal, which presented fever $\left(41^{\circ} \mathrm{C}\right)$ and dehydration, and a cub in pain, as characterized by dorsal arching (Table 1). This cub died on D14 p.i. and was necropsied. At necropsy, mandibular, submandibular, and retropharyngeal lymph nodes were engorged. Fluid was also observed in the thoracic and abdominal cavities as well as hemorrhagic pulmonary lobes and kidneys.

\section{Haematological and biochemical parameters}

Two animals (cub and young) from Group C presented leukocytosis $\left(19,400 / \mathrm{mm}^{3}\right.$ and $\left.15,800 / \mathrm{mm} 3\right)$. However, neutrophilia (72\%) was observed in the cub but not the young animal.

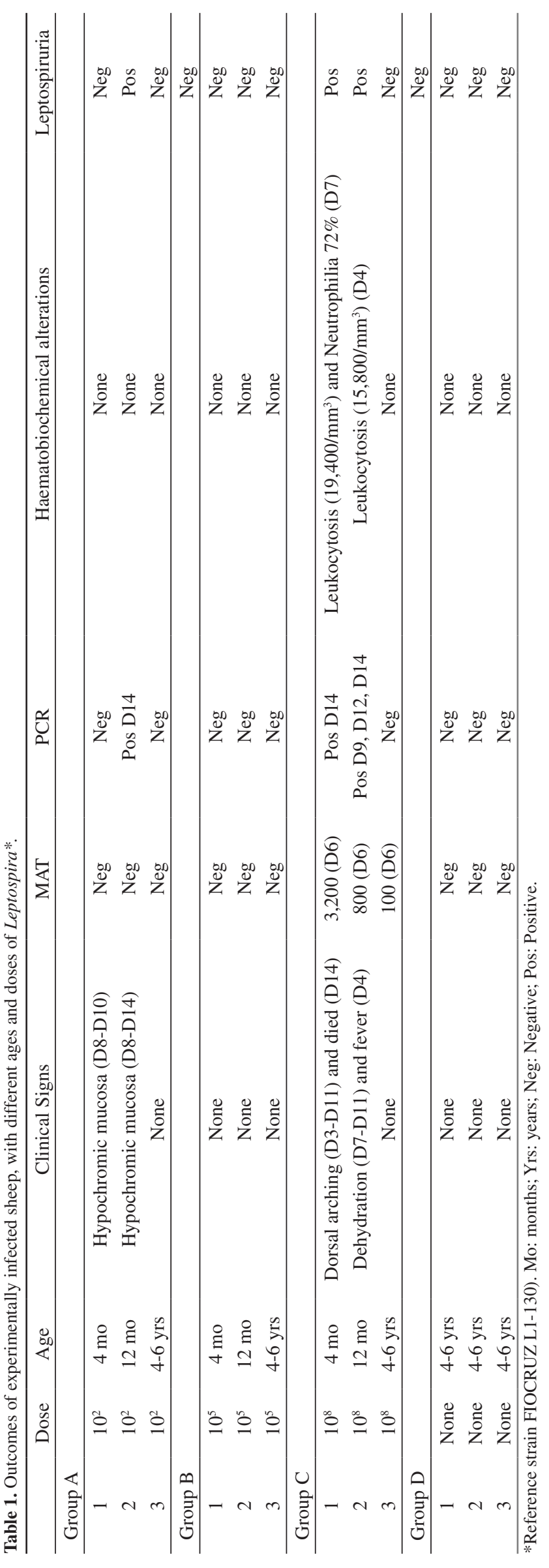


Serological and PCR outcomes

Only the three animals from Group C exhibited positivity in MAT. Titres were observed after D6 and reached up to 3,200 (cub), 800 (young), and 100 (mature). Positivity to PCR was observed in two experimental groups, $\mathrm{A}$ and $\mathrm{C}$, thus confirming the presence of the infection. The young animal from Group C presented positivity on D9, D12, and D14, while the cub of the same group and the young of Group A only showed positivity on D14.

\section{DISCUSSION}

In the present study, experimental infection was successfully achieved in two groups (A and C), as defined by observation of leptospiruria. It was unsurprising that the animals that received the highest dose $\left(10^{8}\right)$ presented more consistent outcomes. These findings corroborate with similar results of studies conducted in New Zealand [14] and Australia [4].

Despite the use of a highly virulent strain, clinical signs of acute leptospirosis were not observed, with the exception of two animals. This was an unexpected and quite frustrating outcome because it is contradictory to other studies that have reported acute clinical signs after experimental infection with highly virulent Leptospira strains [10,11]. Thus, in light of the results of the current study, clinical signs cannot be considered to represent a reliable parameter for evaluating the development of leptospirosis in experimentally infected sheep. It is particularly important to highlight that the age of the animals did not appear to alter their susceptibility to the experimental infection. This finding also contrasts with those of other experimental studies $[4,13]$, which suggested younger animals are more susceptible to acute infection than older ones.

Studies have demonstrated that in sheep the acute form of this disease occurs mainly in European breed animals $[10,14]$. A rustic Brazilian breed (Santa Ines) was tested in the current study. This breed is adapted to adverse conditions, such as drought and food shortage, and has been reported to be particularly resistant to parasitism [1]. As such, there is a strong possibility that the rusticity of the animals influenced the outcomes of the experiment. Although further studies are required, this may represent an interesting research line regarding the identification of the genetic markers involved in any resistance observed.

Regarding the haemato-biochemical parameters, $\mathrm{CBC}$ is the only tool that has proven to be adequate for monitoring experimental infection in sheep. Therefore, since no clinical signs were visible in the current study, it was concluded that daily CBC should be employed as the main tool for assessing infected sheep. CBC has been used to follow the acute phase of leptospirosis, and it is effective in the detection of anemia and leukocytosis with neutrophilia in ruminants [15]. It is important to highlight that alterations to CBC occurred only in animals that received the highest dose of leptospires. Although the majority of the animals did not present leukocytosis in the current study, we did observe a slight variation in this parameter, mainly during the first days of the experiment. However, biochemical alterations were not deemed to be reliable indications of the infectious status, and this finding corroborates with those of other studies $[15,19]$.

Despite the lack of clinical signs, serological and molecular results confirmed the experimental infection. Seroconversion was observed only in the animals that received the highest dose of leptospires (Group C), similar to the findings of previous research $[11,13,14]$. Additionally, in this group, only animals with high titers also presented leptospiruria. PCR was positive in animals from two experimental groups. PCR has been used as an important diagnostic tool for assessing leptospirosis in different species, including sheep [3,6]; however, to the best of our knowledge, the current study is the first of its kind to use PCR to follow up the carrier status in experimentally infected sheep. Nevertheless, the limited number of positive reactions in the present study may be related to the intermittent shedding of leptospires in the urine [5]. Despite that limitation, PCR proved to be very effective in the confirmation of the infection, and it should be considered for use in experimental studies.

Sheep have been used as a good experimental model in several studies, such as in cardiovascular, respiratory, reproductive and, especially, immunology domains [18]. Sheep are relatively small in comparison to other ruminants, such as cattle, and can be easily allocated to smaller pens and sheds, facilitating the management of the research and minimizing the costs of experimentation [24]. Cattle and sheep present a strong chromosome homology, which may justify the similarities in the immune responses they exhibit [17]. It has been suggested that sheep and 
cattle shared an ancestral chromosome that molded the MHC of ruminants and, as such, they potentially share the same induction of immune response [7]. Additionally, Toll-like receptors 1-10 are present in both bovine and ovine tissues during the regulatory stage of the immune response [17]. In this context, we suggest sheep represent a good model for studying leptospirosis in ruminants and, therefore, a reliable protocol for experimental leptospiral infection is required.

\section{CONCLUSION}

In conclusion, we demonstrated that sheep may be experimentally infected and used as a model for leptospirosis in ruminants. As clinical signs were not evident, we recommend urine PCR and serology (MAT) for confirmation of the infection and daily CBC as a follow-up tool to assess experimentally infected animals.

\section{MANUFACTURERS}

${ }^{1}$ Ouro Fino Saúde Animal. Cravinhos, Cravinhos, SP, Brazil.

${ }^{2}$ BD - Becton Dickinson. São Paulo, SP, Brazil.

${ }^{3}$ MSD Saúde Animal Brasil. São Paulo, SP, Brazil.

${ }^{4}$ Institut Pasteur. Paris, France.

${ }^{5}$ Promega Corporation. Madison, MI, USA.

${ }^{6}$ NewProv Prod P Lab. Pinhais, PR, Brazil.

${ }^{7}$ Labtest Diagnóstica S.A. Belo Horizonte, MG, Brazil.

Acknowledgments. This work was supported by CNPq (Grant number 402667/2013). It should be emphasized that in the present study the authors Matheus Costa da Rosa and Bruno Ribeiro Rocha had an equal dedication and contribution to the accomplishment of the project and obtaining the results.

Ethical approval. The study was conducted with the full approval of the Ethics Committee of Universidade Federal Fluminense, Brazil (number 814/2016).

Declaration of interest. The authors report no conflicts of interest. The authors alone are responsible for the content and writing of the paper.

\section{REFERENCES}

1 Amarante A.F., Susin I., Rocha R.A., Silva M.B., Mendes C.Q. \& Pires A.V. 2009. Resistance of Santa Ines and crossbred ewes to naturally acquired gastrointestinal nematode infections. Veterinary Parasitology. 165: 273-280.

2 Andreani E., Tolari F. \& Farina R. 1983. Experimental infection in sheep with Leptospira interrogans serotype hardjo. Brazilian Veterinary Journal. 139: 165-170.

3 Director A., Martins G.M.S., Loureiro A.P.P., Hamond C., Reis R.M., Medeiros M.A. \& Lilenbaum W. 2014. Molecular detection of leptospiral carriers in sheep under tropical field conditions. Brazilian Journal Veterinary Research Animal Science. 51: 220-223.

4 Durfee P.T. \& Presidente P.J. 1979. Experimental infection of calves and sheep with Leptospira interrogans serovar balcanica. Australian Journal Experimental Biology Medical Science. 57: 447-453.

5 Ellis W.A. 2015. Animal leptospirosis. In: Current Topics in Microbiology and Immunology. 387: 99-137.

6 Gamage C.D., Koizumi N., Muto M., Nwafor-Okoli C., Kurukurusuriya S., Rajapakse J.R., Kularatne S.A., Kanda K., Lee R.B., Obayashi Y. \& Watanabe H. \& Tamashiro H. 2011. Prevalence and carrier status of leptospirosis in smallholder dairy cattle and peridomestic rodents in Kandy, Sri Lanka. Vector-Borne and Zoonotic Diseases. 11: 1041-1047.

7 Gao J., Liu K., Blair H.T., LI G., Chen C., Tan P. \& Ma R.Z. 2010. A complete DNA sequence map of the ovine Major Histocompatibility Complex. BMC Genomics. 11: 466.

8 Hamond C., Martins G., Loureiro A.P., Pestana C., Lawson-Ferreira R., Medeiros M.A. \& Lilenbaum W. 2014. Urinary PCR as an increasingly useful tool for an accurate diagnosis of leptospirosis in livestock. Veterinary Research Communications. 38: 81-85.

9 Hathaway S.C. \& Marshall R.B. 1979. Experimental infection of sheep with Leptospira interrogans: serovars hardjo and balcanica. New Zealand Veterinary Journal. 27: 197.

10 Hodges R.T. 1974. Some observations on experimental Leptospira serotype Pomona infection in sheep. New Zealand Veterinary Journal. 22: 151-154.

11 Langham R.F., Morse E.V. \& Morter R.L. 1958. Pathology of experimental ovine leptospirosis, Leptospira pomona infection. Journal of Infectious Diseases. 103: 285-290.

12 Martins G., Penna B. \& Lilenbaum W. 2012. Differences between seroreactivity to leptospirosis in dairy and beef cattle from the same herd in Rio de Janeiro, Brazil. Tropical Animal Health and Production. 44: 377-378.

13 Mackintosh C.G., Marshall R.B. \& Thompson J.C. 1981. Experimental infection of sheep and cattle with Leptospira interrogans serovar balcanica. New Zealand Veterinary Journal. 29: 15-19. 
14 Marshall R.B., Broughton E.S. \& Hathaway S.C. 1979. Protection of sheep by vaccination against artificial challenge with Leptospira interrogans serovar hardjo. New Zealand Veterinary Journal. 27: 195-196.

15 Nervig R.M., Cheville N.F. \& Baetz A.L. 1978. Experimental infection of calves with Leptospira interrogans serotype szwajizak. American Journal of Veterinary Research. 39: 523-255.

16 OIE. 2014. Manual of Diagnostic Tests and Vaccines for Terrestrial Animals. 7th edn. Paris: W. Organism for Animal Health. 1: 1343

17 Perucatti A., Genualdo D., Iannuzzi A., Rebl A., Di Berardino D., Goldammer T. \& Iannuzzi L. 2012. Advanced comparative cytogenetic analysis of X chromosomes in river buffalo, cattle, sheep, and human. Chromosome Research. 20: $413-425$

18 Ramos J.J., Lacasta D., Ferrer L.M. \& de Arcaute M.R. 2012. A novel educational approach: Using sheep as a model in teaching veterinary pathology. Small Ruminant Research. 110: 133-137

19 Ristic M., Galton M.M., Mcrae L., Sanders D.A. \& Steele J.H. 1957. Experimental leptospirosis in bovines. I. Establishment of infection with Leptospira sejroe. Journal of Infectious Disease. 100: 228-240

20 Ridpath J.F., Falkenberg S.M., Bauermann F. V., VanderLey B.L., YoonJung Do, Flores E.F., Rodman D.M. \& Neill J.D. 2013. Comparison of acute infection of calves exposed to a high virulence or low virulence bovine viral diarrhea virus or a Hobi-like virus. American Journal Veterinary Research. 74: 438-442

21 Ridzuan J.M., Aziah B.D. \& Zahiruddin W.M. 2016. Study on Seroprevalence and Leptospiral Antibody Distribution among High-risk Planters in Malaysia. Osong Public Health and Research Perspectives. 7: 168-171.

22 Salviano M.B, Sousa Junior A. \& Moura W.L. 2013. Hematologic Sheep of Santa Ines Adults Not Pregnant. Revista Científica Eletrônica de Medicina Veterinária. 20: 1-12

23 Schneider M.C., Jancloes M., Buss D.F., Aldighieri S., Bertherat E., Najera P., Galan D.I., Durski K. \& Espinal M.A. 2013. Leptospirosis: a silent epidemic disease. International Journal of Environmental Research and Public Health. 16: 7229-7234.

24 Scheerlinck J.P.Y., Snibson K.J., Bowles V.M. \& Sutton P. 2008. Biomedical applications of sheep models: from asthma to vaccines. Trends on Biotechnology. 26: 259-266.

25 Suepaul S.M., Carrington C.V., Campbell M., Borde G. \& Adesiyun A.A. 2011. Seroepidemiology of leptospirosis in livestock in Trinidad. Tropical Animal Health Production. 43: 367-375.

26 Thrall M.A. \& Weiser M.G. 2006. Procedimentos laboratoriais para técnicos veterinários. In: Hematologia hendrix. (São Paulo, Brasil). pp.31-78.

27 Vihol P.D., Patel J.M., Patel J.H., Prasad M.C., Kalyani I.H. \& Brahmkshtri B.P. 2016. Caprine leptospirosis: Hematobiochemical and urinalyses studies. Veterinary World. 9: 337-341. 